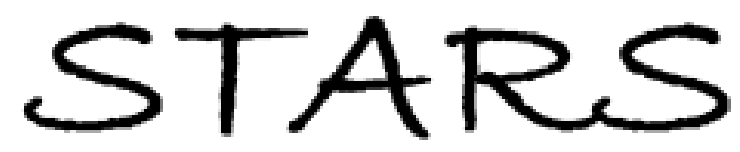

University of Central Florida

STARS

$1-1-2013$

\title{
A low voltage and submillisecond-response polymer-stabilized blue phase liquid crystal
}

\author{
Yuan Chen \\ University of Central Florida \\ Daming $\mathrm{Xu}$ \\ University of Central Florida \\ Shin-Tson Wu \\ University of Central Florida \\ Shin-ichi Yamamoto \\ Yashuhiro Haseba
}

Find similar works at: https://stars.library.ucf.edu/facultybib2010

University of Central Florida Libraries http://library.ucf.edu

This Article is brought to you for free and open access by the Faculty Bibliography at STARS. It has been accepted for inclusion in Faculty Bibliography 2010 s by an authorized administrator of STARS. For more information, please contact STARS@ucf.edu.

\section{Recommended Citation}

Chen, Yuan; Xu, Daming; Wu, Shin-Tson; Yamamoto, Shin-ichi; and Haseba, Yashuhiro, "A low voltage and submillisecond-response polymer-stabilized blue phase liquid crystal" (2013). Faculty Bibliography 2010s. 3797.

https://stars.library.ucf.edu/facultybib2010/3797

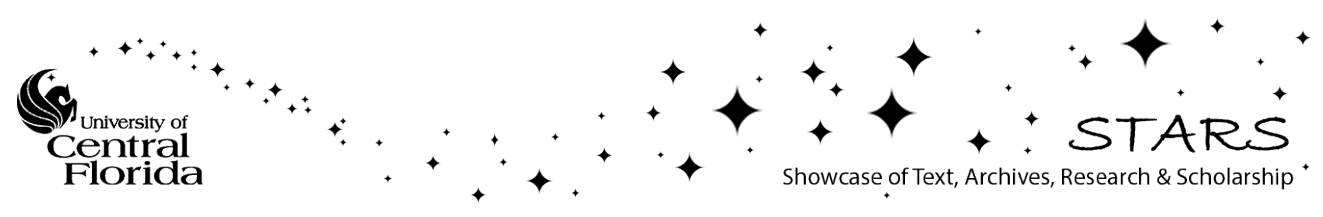




\section{A low voltage and submillisecond-response polymer-stabilized blue phase liquid crystal}

Cite as: Appl. Phys. Lett. 102, 141116 (2013); https://doi.org/10.1063/1.4802090

Submitted: 13 March 2013 . Accepted: 02 April 2013 . Published Online: 12 April 2013

Yuan Chen, Daming Xu, Shin-Tson Wu, Shin-ichi Yamamoto, and Yasuhiro Haseba
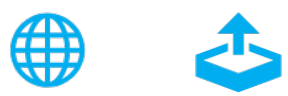

\section{ARTICLES YOU MAY BE INTERESTED IN}

Electro-optics of polymer-stabilized blue phase liquid crystal displays

Applied Physics Letters 94, 101104 (2009); https://doi.org/10.1063/1.3097355

A large Kerr constant polymer-stabilized blue phase liquid crystal

Applied Physics Letters 98, 081109 (2011); https://doi.org/10.1063/1.3559614

Low voltage blue-phase liquid crystal displays

Applied Physics Letters 95, 231101 (2009); https://doi.org/10.1063/1.3271771

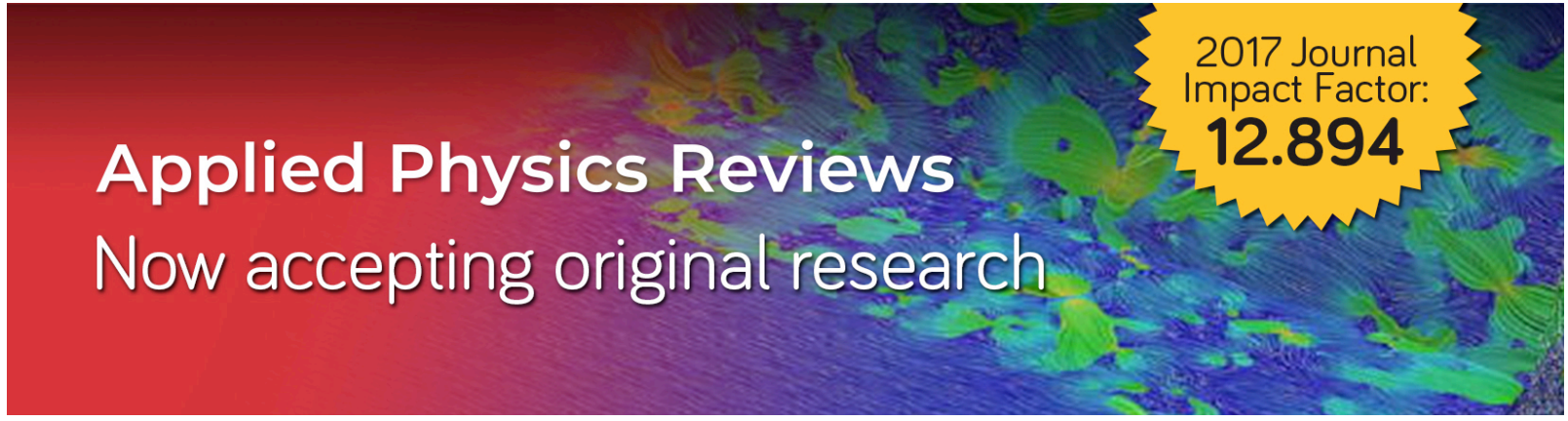




\title{
A low voltage and submillisecond-response polymer-stabilized blue phase liquid crystal
}

\author{
Yuan Chen, ${ }^{1}$ Daming Xu, ${ }^{1}$ Shin-Tson Wu, ${ }^{1, a)}$ Shin-ichi Yamamoto, ${ }^{2}$ and Yasuhiro Haseba ${ }^{2}$ \\ ${ }^{1}$ College of Optics and Photonics, University of Central Florida, Orlando, Florida 32816, USA \\ ${ }^{2}$ JNC Petrochemical Corporation, Ichihara Research Center, Ichihara, Chiba 290-8551, Japan
}

(Received 13 March 2013; accepted 2 April 2013; published online 12 April 2013)

\begin{abstract}
We report a polymer-stabilized blue phase liquid crystal (BPLC) whose Kerr constant is about $2.2 \times$ larger than previous record. When filled in a 3.2- $\mu$ m-thick vertical field switching cell, the on-state voltage is merely $8.4 \mathrm{~V}$ (at $\lambda=514 \mathrm{~nm}$ ) while keeping submillisecond response time and negligible hysteresis $(<1 \%)$ at the room temperature. These results imply that the dawn of BPLC era for high speed display and photonic devices has finally arrived. (C) 2013 AIP Publishing LLC [http://dx.doi.org/10.1063/1.4802090]
\end{abstract}

Polymer-stabilized blue-phase liquid crystal (PS-BPLC) $)^{1-5}$ exhibits three distinctive features: (1) self-assembly process so that no surfactant (e.g., rubbed polyimide layer) is needed to generate uniform molecular alignment, (2) nanoscale ( $\sim 100 \mathrm{~nm})$ double-twist cylinder diameter and short coherence length, which leads to microsecond response time, ${ }^{6,7}$ and (3) three-dimensional lattice structure resulting in optically isotropic voltage-off state. Alignment-layer-free process greatly simplifies device fabrication process. Fast response time not only produces crisp pictures without image blurs but also enables color sequential displays. ${ }^{8-10}$ By eliminating spatial color filters, both optical efficiency and resolution density are tripled. This feature is particularly important for reducing the power consumption of a high resolution retina display. Moreover, short coherence length significantly improves the diffraction efficiency of BPLC gratings. ${ }^{11}$ Finally, optically isotropic state makes adaptive BPLC lenses polarization independent. ${ }^{12,13}$ Therefore, BPLC holds promises for display ${ }^{4,14}$ and photonics applications. ${ }^{15}$

However, high operation voltage, noticeable hysteresis, and large capacitance still hinder its widespread applications. Among these three technical barriers, high voltage is the root cause. If the voltage is low $(<10 \mathrm{~V})$, then the electrostriction effect, which causes lattice distortion would be prohibited and hysteresis be negligible $(<1 \%)$, and high frame rate $(>180 \mathrm{~Hz})$ can be achieved by the bootstrapping circuits. ${ }^{16}$ Therefore, the most fundamental issue for BPLC studies is to lower the operation voltage to below $10 \mathrm{~V}$, without sacrificing other desirable properties, such as high transmittance, submillisecond response time, high contrast ratio $(\mathrm{CR})$, and wide viewing angle. To achieve this goal, both device structures and BPLC materials have been investigated extensively. From device structure viewpoint, two major approaches have been developed: (1) implementing protrusion electrodes so that the fringing field can penetrate deeply into the LC bulk, ${ }^{17-19}$ and (2) vertical field switching (VFS) ${ }^{20}$ to generate uniform longitudinal field across the BPLC layer. From material aspect, developing BPLC materials with a large Kerr constant $(K)^{21,22}$ helps reduce driving voltage because the on-state voltage is inversely proportional to $\sqrt{K} .{ }^{17}$ From

a)E-mail: swu@ucf.edu
Gerber's model, Kerr constant is governed by the birefringence $(\Delta n)$, dielectric anisotropy $(\Delta \varepsilon)$, average elastic constant $(k)$, and pitch length $(p)$ of the chiral LC host as ${ }^{23}$

$$
K \sim \Delta n \cdot \Delta \varepsilon \cdot p^{2} / k
$$

As Eq. (1) indicates to enlarge $K$ by increasing pitch length seems to be a straightforward approach because $K$ is proportional to $p^{2}$. However, this approach has its own limitations, as reported by Yan et al. ${ }^{24}$ Therefore, it is better to keep Bragg reflection in the UV region $(\sim 350 \mathrm{~nm})$. From Eq. (1), the remaining approach is to boost $\Delta \mathrm{n} \cdot \Delta \varepsilon$ because $k$ is more difficult to control. ${ }^{3}$ The LC birefringence is mainly determined by the conjugation length. Considering the material stability, melting temperature, and viscosity, terphenyl derivatives could be the longest conjugation for BPLCs. Therefore, increasing $\Delta \varepsilon$ becomes a popular option. Indeed, LC hosts with $\Delta \varepsilon(>90)$ have been developed by $\mathrm{JNC}^{21}$ and Merck $^{22}$ independently. Such a large $\Delta \varepsilon$ LC would require several polar groups, so that its rotational viscosity and response time would increase dramatically. Indeed, the response time of $\mathrm{JC}-\mathrm{BP} 01 \mathrm{M}$ is about $2 \mathrm{~ms}$ at $\mathrm{RT} \sim 22^{\circ} \mathrm{C}$ using an in-plane-switching (IPS) cell. ${ }^{21}$ In addition, the hysteresis is about $6 \%$ because of the strong peak electric field near the IPS electrode edges. ${ }^{25}$ A delicate balance between operating voltage, response time, and hysteresis has to be taken into consideration.

In this Letter, we report a record-high Kerr constant PSBPLC while keeping submillisecond response time and negligible hysteresis at room temperature. The measured Kerr constant is $33.1 \mathrm{~nm} / \mathrm{V}^{2}$ in an IPS cell and $20.9 \mathrm{~nm} / \mathrm{V}^{2}$ in a VFS cell at $\lambda=514 \mathrm{~nm}$. The on-state voltage of our VFSBPLC device is $8.4 \mathrm{~V}_{\mathrm{rms}}$ at $\lambda=514 \mathrm{~nm}$, hysteresis $0.9 \%$, and response time $<1 \mathrm{~ms}$ at $\mathrm{RT}$.

In the experiment, we prepared a large $\Delta \varepsilon$ nematic LC host JC-BP06N (by JNC) whose clearing point is $T_{c}=73.8^{\circ} \mathrm{C}$. We filled JC-BP06N into a homogeneous cell with cell gap $d \sim 5 \mu \mathrm{m}$. The cell has strong anchoring energy and about $3^{\circ}$ pretilt angle. To evaluate the birefringence of the LC host, we measured the phase retardation of a homogeneous cell sandwiched between two crossed polarizers. ${ }^{26}$ However, this LC host does not align well in the homogeneous cell. As shown in 


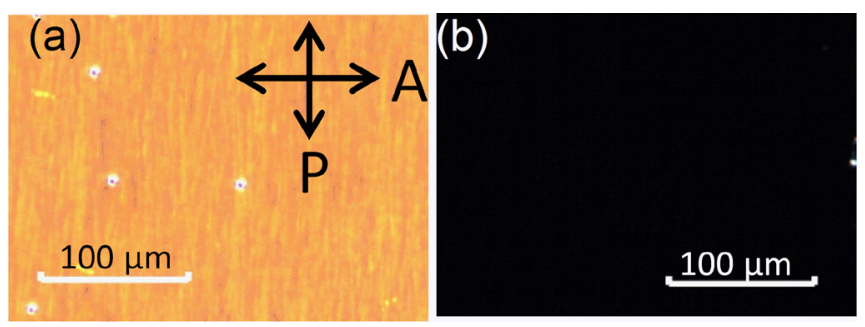

FIG. 1. POM images of the homogeneous cells filled with (a) JC-BP06N and (b) LC1 (with alignment direction parallel to the optic axis of the polarizer)

Fig. 1(a), when the LC alignment direction is parallel to the optic axis of the polarizer, the cell shows severe light leakage (under white light) between crossed polarizers, indicating the LC molecules do not follow the rubbing direction well. Therefore, we could not determine $\Delta n$ accurately from the cell due to poor alignment. Similar alignment problem of negative $\Delta \varepsilon$ LCs in vertically aligned (VA) cells has been reported before, and diluters can be used to improve the alignment. ${ }^{27}$ Therefore, we doped $5 \mathrm{wt}$. \% diluter: 4-pentyl-4'-propyl1,1'-bi(cyclohexyl) (5CC3) into JC-BP06N. For convenience, let us call this mixture $\mathrm{LC} 1$; its $T_{c}$ is $74{ }^{\circ} \mathrm{C}$. LC1 shows good alignment in the homogeneous cell and it appears dark under crossed polarizers [Fig. 1(b)] when the alignment direction is parallel to the optic axis of the polarizer. We then measured the voltage-dependent transmittance (VT) of the LC1 cell and obtained $\Delta n=0.156$ at $\lambda=514 \mathrm{~nm}$ and room temperature $\left(\mathrm{RT}=22^{\circ} \mathrm{C}\right)$. Through the relaxation time measurement of the LC1 cell, ${ }^{28}$ we obtained the viscoelastic constant $\gamma_{1} / k_{11}=199 \mathrm{~ms} / \mu \mathrm{m}^{2}$ and rotational viscosity $\gamma_{1}=1.47$ Pas. This $\gamma_{1} / k_{11}$ is about $10 \times$ larger than that of nematic E7 mixture. Diluter tends to decrease $\Delta n$ of the large $\Delta \varepsilon$ LC slightly but decrease $\gamma_{1}$ dramatically. Therefore, JC-BP06N should have a larger $\gamma_{1}$ and slightly higher $\Delta n(\sim 0.17)$ than LC1.

Dielectric anisotropy of a LC is defined as $\Delta \varepsilon=\varepsilon_{/ /}^{\prime}-\varepsilon_{\perp}^{\prime}$, here $\varepsilon_{/ /}^{\prime}$ and $\varepsilon_{\perp}^{\prime}$ represent the real part of the parallel and perpendicular dielectric permittivity. To determine the $\Delta \varepsilon$ of these two LCs, we measured the capacitance of a homogeneous cell and a VA cell using an HP-4274 multi-frequency LCR meter. ${ }^{28}$ Both LCs align well in the VA cell. However, the poor alignment of JC-BP06N in the homogeneous cell should result in a larger $\varepsilon_{\perp}^{\prime}$ than the actual value. The measured $\varepsilon_{\perp}^{\prime}$ of JC-BP06N is 17.9 at $100 \mathrm{~Hz}$, which is much smaller than $\varepsilon_{/ /}^{\prime}(491)$, therefore the error in $\varepsilon_{\perp}^{\prime}$ will not affect $\Delta \varepsilon$ too much. Figure 2 depicts the measured results. The solid and open circles are the measured $\varepsilon_{/ /}^{\prime}$ for JC-BP06N and LC1, respectively, and the lines are fitting results with Debye relaxation equation $^{29,30}$

$$
\varepsilon_{/ /}^{\prime}=\varepsilon_{\infty}+\frac{\varepsilon_{0}-\varepsilon_{\infty}}{1+\left(f / f_{r}\right)^{2}},
$$

where $\varepsilon_{0}$ is the static permittivity along the long molecular axis at the low frequency limit, and $\varepsilon_{\infty}$ is the permittivity at the high frequency limit. From these data, we obtain $\varepsilon_{0}=491$ at low frequency region for JC-BP06N. As the frequency increases, $\varepsilon_{/ /}^{\prime}$ decreases dramatically due to the slow dielectric relaxation time resulting from the large viscosity and bulky molecular structure. ${ }^{31}$ Through fitting with Eq. (2), we find

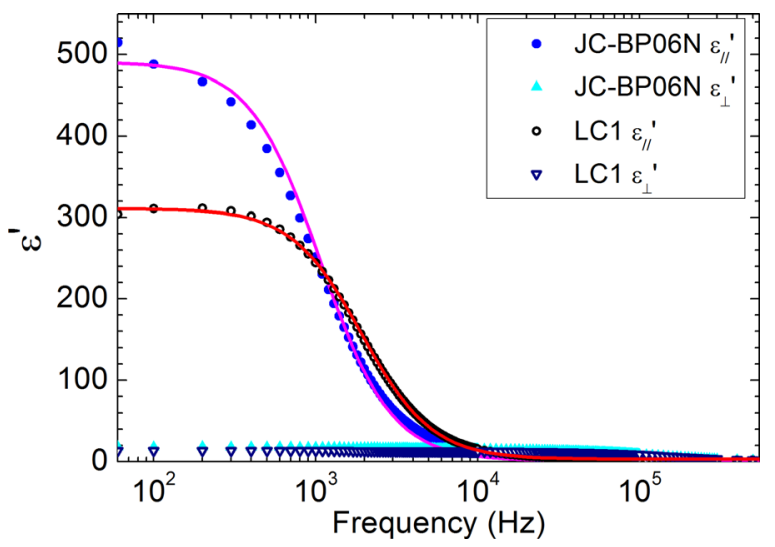

FIG. 2. Frequency-dependent $\varepsilon_{/ /}^{\prime}$ and $\varepsilon_{\perp}^{\prime}$ of JC-BP06N and LC1.

$f_{r}=1.07 \mathrm{kHz}$. For conventional nematic LCs, the relaxation frequency is usually quite high $(>50 \mathrm{kHz})$. After doping a small amount of diluter, the $f_{r}$ of LC1 shifts to $1.92 \mathrm{kHz}$ because of the dramatically reduced viscosity. ${ }^{32}$ However, $\varepsilon_{0}$ drops significantly from 491 to 311 after adding $5 \mathrm{wt}$. \% $5 \mathrm{CC} 3$. The small molecules of 5CC3 are uniformly distributed in JC-BP06N. According to Lichtenecker formula, the dielectric constant $\varepsilon_{m}$ of a binary mixture of liquids can be expressed as ${ }^{33}$

$$
\varepsilon_{m}=\exp \left(\varepsilon_{1} \ln \left(\Omega_{1}\right)+\varepsilon_{2} \ln \left(1-\Omega_{1}\right)\right),
$$

where $\varepsilon_{1}$ and $\varepsilon_{2}$ are the parallel dielectric constant of the two components and $\Omega_{l}$ is the volume fraction of component 1 . If we substitute $\varepsilon_{1}=2.35$ (measured data for 5CC3), $\varepsilon_{2}=491$, and $\Omega_{1}=0.05$ (wt. \%) into Eq. (3), we find $\varepsilon_{m}=376$. However, $\Omega_{l}$ should be larger than the wt. \% because of the smaller molecular weight of 5CC3, compared to JC-BP06N. If we assume $\Omega_{l}=0.085$, then we obtain $\varepsilon_{m}=312$, which is quite close to the measured result $(\sim 311)$.

Although we are able to get good alignment and measure the physical properties of LC1, LC1 has much smaller $\Delta \varepsilon(298$ vs. 473) and will result in much smaller $K$ than JCBP06N. Therefore, we prepared a PSBP sample employing JC-BP06N. The blue phase precursor consists of $88.17 \mathrm{wt} \%$ JC-BP06N with 2.92 wt. \% of chiral dopant R5011 (HCCH), 5.24 wt. \% RM257 (Merck), 3.46 wt. \% TMPTA (1,1, 1-Trimethylolpropane Triacrylate, Sigma Aldrich) and 0.21 wt. \% photoinitiator. Next, we injected the LC/monomers mixture into a VFS cell ${ }^{20}$ in an isotropic phase. The VFS cell is comprised of two ITO (indium tin oxide) glass substrates, but without polyimide alignment layer. The cell gap is controlled at $\sim 3.2 \mu \mathrm{m}$. Afterwards, the cell was placed on a Linkam heating/freezing stage controlled by a temperature programmer (Linkam TMS94). The cell was cooled to blue phase and then exposed to UV light $(\lambda \sim 365 \mathrm{~nm})$ with intensity of $2 \mathrm{~mW} / \mathrm{cm}^{2}$ for $30 \mathrm{~min}$. After UV irradiation, nano-structured BPLC composites were self-assembled. The measured Bragg reflection wavelength is $\sim 380 \mathrm{~nm}$, which is about the same as that of JC-BP01M (our benchmarking BPLC material).

The experimental setup for characterizing the electro-optic properties of a VFS cell has been reported previously. ${ }^{20} \mathrm{~A}$ linearly polarized Argon ion laser $(\lambda=457 \mathrm{~nm}$ and $\lambda=514 \mathrm{~nm})$ and a He-Ne laser $(\lambda=633 \mathrm{~nm})$ were used as probing beams. 
In order to acquire phase retardation, the laser beam was incident on the VFS cell at $70^{\circ}$ oblique angle. The VFS cell was immersed in a transparent container filled with Glycerol $(n=1.47)$, so that the beam could enter the LC layer at a large angle due to the refractive index match between the glass and Glycerol. The container was placed between two crossed polarizers, and the light transmittance was measured by a photodiode detector (New Focus Model 2031) and recorded digitally by a LABVIEW data acquisition system (DAQ, PCI 6110).

In order to compare the electro-optic properties of our PS-BPLC to JC-BP01M, ${ }^{21}$ we measured our cell using the same driving frequency $100 \mathrm{~Hz}$. As shown in Fig. 2, the $\Delta \varepsilon$ of JC-BP06N decreases as frequency increases. Accordingly, the Kerr constant will decrease following the extended Coles-Coles model ${ }^{34}$ Ideally, a driving frequency higher than $180 \mathrm{~Hz}$ is required for color sequential displays. If we increase the driving frequency to $240 \mathrm{~Hz}$, Kerr constant will drop by $8 \%$, which in turn increases the on-state voltage by $\sim 4 \%$. This prediction is validated experimentally.

Figure 3(a) depicts the measured VT curve at different wavelengths. Here, the transmittance is normalized to that of two parallel polarizers. From Fig. 3(a), we find the on-state voltage (corresponding to peak transmittance) is $V_{\text {on }}=7.3$, 8.4 , and $9.9 \mathrm{~V}_{\mathrm{rms}}$ for $\lambda=457,514$, and $633 \mathrm{~nm}$, respectively. In the voltage-off state, blue phase is not perfectly optically isotropic but with a tiny optically rotatory power. ${ }^{35}$ At $\lambda=514 \mathrm{~nm}$, our measured CR is 580:1 under crossed polarizers. If we rotate the analyzer by $1.8^{\circ}, \mathrm{CR}$ is boosted to 1900:1. It can be further improved by shifting the Bragg reflection wavelength to the shorter wavelength side, but the operating voltage will increase. The depolarization from the oblique incidence might also degrade CR. As voltage increases, the induced birefringence increases as described by the following extended Kerr model: ${ }^{36}$

$$
\Delta n_{\text {ind }}=\Delta n_{s}\left(1-\exp \left[-\left(E / E_{s}\right)^{2}\right]\right),
$$

where $\Delta n_{s}$ is the saturated induced birefringence and $E_{s}$ is the saturation electric field. Through fitting the VT curves, ${ }^{14}$ we obtained $K=\Delta n_{s} /\left(\lambda E_{s}^{2}\right)=24.9,20.9$, and $17.4 \mathrm{~nm} / \mathrm{V}^{2}$ for $\lambda=457,514$, and $633 \mathrm{~nm}$, respectively. For comparison, at

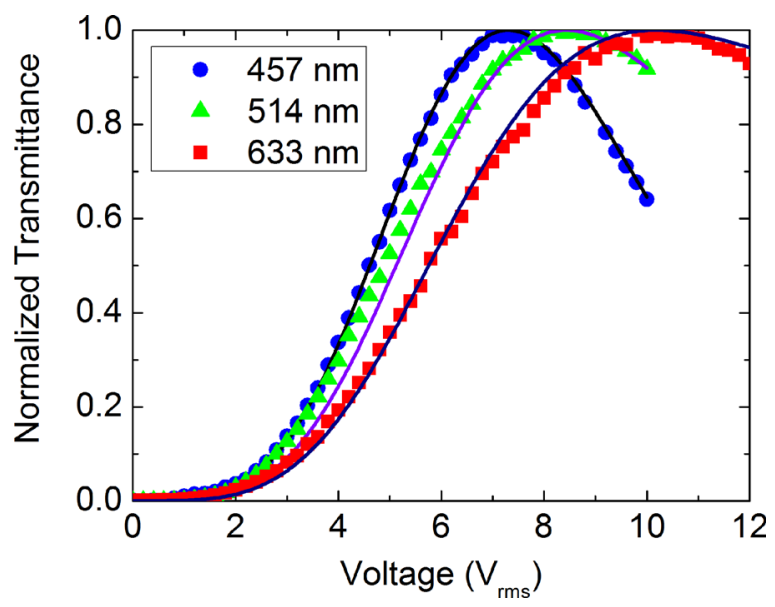

FIG. 3. VT curves of the VFS cell at the specified wavelengths. Dots are measured data and lines are fitted curves with extended Kerr model. Cell gap $d=3.2 \mu \mathrm{m}$. $\lambda=633 \mathrm{~nm}$, the Kerr constant of our BPLC is $\sim 2.3 \times$ larger than that of JC-BP01M (also from VFS cell). We also studied the IPS cells and results will be discussed later.

Next, we measured the rise time and decay time of the VFS cell by swinging the voltage between 0 and $V_{\text {on }}$. The response time is defined as $90 \%$ to $10 \%$ transmittance change. At $\lambda=514 \mathrm{~nm}$ and room temperature, the rise time is $460 \mu \mathrm{s}$ and decay time is $950 \mu \mathrm{s}$. It is known that the BPLC response time in the low field region where lattice distortion does not occur is primarily governed by the viscosity, average elastic constant, and pitch length. ${ }^{7,23,37}$ Polymer structure and polymer concentration also play important roles. ${ }^{32}$ Although the viscosity of our JC-BP06N is quite high, the monomer (TMPTA) we employed has three functional groups, which provides strong crosslinking networks and helps reduce the response time. A tradeoff is higher voltage.

Hysteresis affects grayscale accuracy of a BPLC device and should be minimized. ${ }^{38}$ To characterize hysteresis, we drove the VFS cell by ascending the voltage to $V_{o n}$ and then gradually descending it to 0 . Hysteresis is defined as $\Delta V / V_{\text {on }}$, where $\Delta V$ is the voltage difference between the forward and backward scans at half of the peak transmittance. For the VFS cell, the measured hysteresis is only $\sim 0.9 \%(\Delta V=0.08 \mathrm{~V})$ at $\lambda=514 \mathrm{~nm}$, which is negligible. If the applied voltage exceeds $8.4 \mathrm{~V}_{\mathrm{rms}}$, then the hysteresis will gradually increase. Here, we define a critical field as $E_{c}=V_{o n} / d$. For the BPLC we studied, its $E_{c}=2.67 \mathrm{~V} / \mu \mathrm{m}$. Above $E_{c}$, hysteresis will gradually grow and response time becomes slower. ${ }^{25,39}$ Therefore, the cell gap of a VFS cell plays an important role affecting the on-state voltage and hysteresis.

For VFS mode, the on-state voltage depends on the cell gap. When a BPLC layer is subject to a longitudinal field, the induced birefringence is along the electric field direction and the cell behaves as a C-plate. ${ }^{40}$ The phase retardation can be described by

$$
\Gamma=\frac{2 \pi}{\lambda} n_{o} d\left[\sqrt{1-\frac{n_{g}^{2} \sin ^{2} \theta}{n_{e}^{2}}}-\sqrt{1-\frac{n_{g}^{2} \sin ^{2} \theta}{n_{o}^{2}}}\right],
$$

where $n_{g}$ is the refractive index of Glycerol, $\theta$ is the incident angle, and $n_{e}$ and $n_{o}$ are the ordinary and extraordinary refractive indices of the BPLC. When $\Gamma(V)=\pi$, we get the peak transmittance and $V_{\text {on }}$ under crossed polarizers. We assume the following equations hold for $n_{e}$ and $n_{o}:{ }^{36}$

$$
\begin{gathered}
n_{o}=n_{i}-\Delta n / 3, \\
n_{e}=n_{i}+2 \Delta n / 3,
\end{gathered}
$$

where $n_{i}$ is the isotropic refractive index of BPLC in the voltage-off state. Combining Eqs. (4) and (6) and plugging the fitting parameters $E_{s}$ and $\Delta n_{s}$, we can obtain $n_{o}, n_{e}$ and hence $\Gamma$ at different voltages. Figure 4 depicts the cell gap effect on $V_{o n}$. A thinner cell gap results in a stronger electric field so that the induced birefringence is larger, which in turn lowers $V_{o n}$. However, as $d$ continually decreases the accumulated phase retardation would be smaller than $1 \pi$. Eventually, $V_{\text {on }}$ will increase as the cell gap gets too thin. When $d$ varies from $2.13 \mu \mathrm{m}$ to $5.9 \mu \mathrm{m}, V_{\text {on }}$ stays below $10 \mathrm{~V}$ for our PS-BPLC. However, to suppress hysteresis, the peak electric 


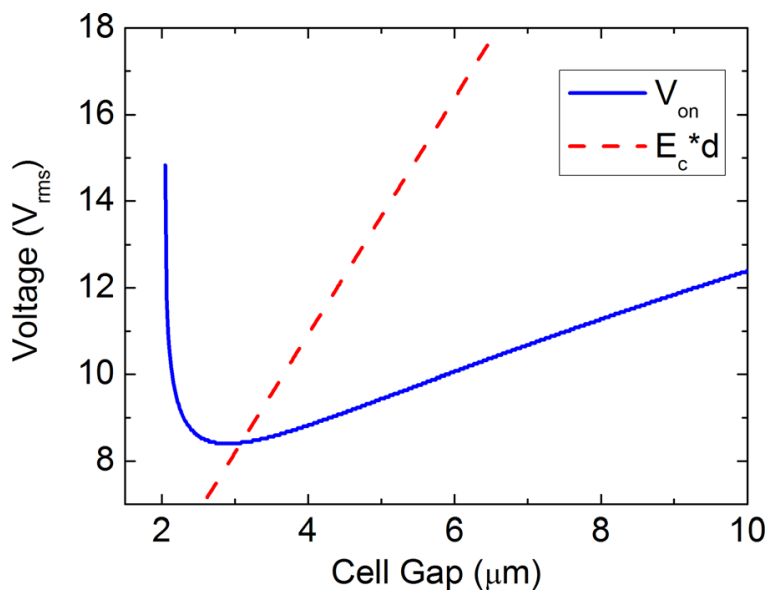

FIG. 4. Cell gap effect on $V_{\text {on }}$ and optimized cell gap for hysteresis-free and $\mathrm{V}_{\text {on }}<10 \mathrm{~V}$.

field should not exceed the critical field $E_{c}$, i.e., $V_{o n}<E_{c} * d$. In Fig. 4, the red dashed lines show the critical voltage $\left(E_{c} * d\right)$ for different cell gaps. To keep hysteresis negligible, the cell gap should be larger than $3.1 \mu \mathrm{m}$. Therefore, the optimized cell gap should be $3.1 \mu \mathrm{m}<\mathrm{d}<5.9 \mu \mathrm{m}$.

The above discussions are all focused on VFS cell, in which the electric field is in longitudinal direction. VFS mode offers several spectacular properties, such as low voltage, high transmittance, fast response time, and negligible hysteresis. However, it requires a directional backlight and sophisticated phase compensation in order to achieve wide view. ${ }^{41}$ Another possible driving method is to use fringing field generated from in-plane switching (IPS) electrodes. The major advantages of IPS mode are twofold: 1) a normal backlight can be used, and 2) wide viewing angle can be obtained by a simple biaxial compensation film. ${ }^{40}$

In experiment, we filled the PS-BPLC to an IPS-5/5 cell: the electrode width and gap are both $5 \mu \mathrm{m}$, and cell gap is $7 \mu \mathrm{m}$. The PSBP stabilization procedure is the same as the VFS cell. After polymer stabilization, the IPS cell was sandwiched between two crossed polarizers and the voltage dependent transmitted light was focused by a lens, so that different diffraction orders ${ }^{11}$ can be collected by the detector. The peak transmittance of the IPS-5/5 cell is about $60 \%$ because of the dead zones on top of the electrodes. Figure 5

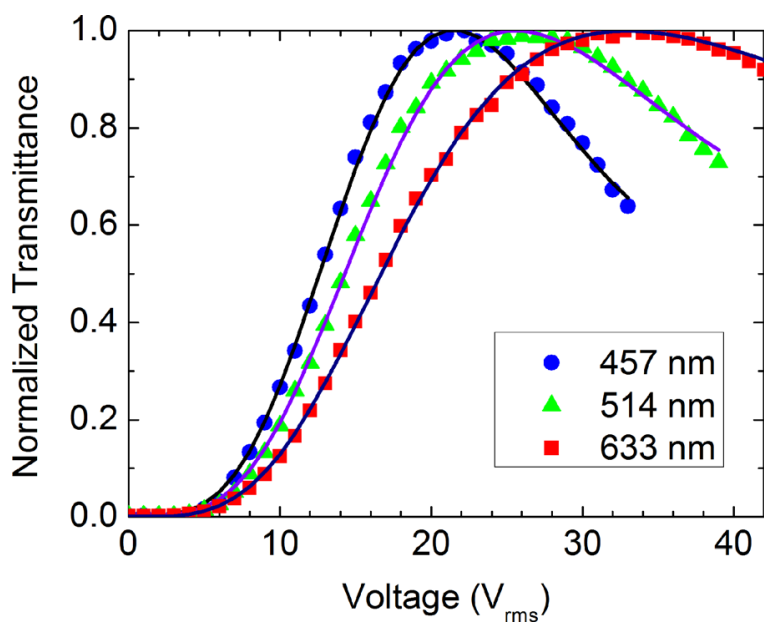

FIG. 5. The VT curves (data and fitting) at the specified wavelengths of an IPS-5/5 cell. is a plot of the normalized transmittance at different wavelengths. From Fig. 5, we find $V_{\text {on }}=22.2,25.4$, and $32.8 \mathrm{~V}_{\mathrm{rms}}$ at $\lambda=457,514$, and $633 \mathrm{~nm}$, respectively. Through fitting the data with the extended Kerr model, ${ }^{14,36}$ we get $K=39.7$, 33.1 , and $26.9 \mathrm{~nm} / \mathrm{V}^{2}$, correspondingly. These Kerr constants are larger than those obtained from VFS cell because of the nonuniform electric field distribution in the IPS cell. By contrast, the Kerr constant of our BPLC is $\sim 2.1 \times$ larger than that of JC-BP01M at room temperature (also in IPS cell). ${ }^{21}$ This improvement is close to $2.3 \times$ by using the VFS cells, considering the measurement and fitting errors. So, on average, our PS-BPLC shows a $2.2 \times$ larger Kerr constant than JC-BP01M.

In conclusion, we have demonstrated a BPLC host with $\Delta \varepsilon \sim 470$ and estimated $\Delta n \sim 0.17$. Good alignment of this BPLC in homogeneous cells was obtained by doping $\sim 5 \mathrm{wt}$. $\%$ of a diluter so that its physical properties can be estimated. Using such a huge $\Delta \varepsilon$ BPLC in a 3.2- $\mu \mathrm{m}$ VFS cell, we have achieved following outstanding results: operation voltage $\sim 8.4 \mathrm{~V}_{\text {rms }}$, rise time $\sim 460 \mu \mathrm{s}$ and decay time $\sim 950 \mu \mathrm{s}$ at room temperature $\left(\sim 22{ }^{\circ} \mathrm{C}\right)$, and hysteresis $\sim 0.9 \%$. The Kerr constant is about $2.2 \times$ higher than previous record.

The authors are indebted to ITRI (Taiwan) for financial support and providing the IPS cells.

${ }^{1}$ H. Kikuchi, M. Yokota, Y. Hisakado, H. Yang, and T. Kajiyama, Nature Mater. 1, 64 (2002).

${ }^{2}$ Y. Hisakado, H. Kikuchi, T. Nagamura, and T. Kajiyama, Adv. Mater. 17, 96 (2005).

${ }^{3}$ S. W. Choi, S. Yamamoto, Y. Haseba, H. Higuchi, and H. Kikuchi, Appl. Phys. Lett. 92, 043119 (2008).

${ }^{4}$ J. Yan, L. Rao, M. Jiao, Y. Li, H. C. Cheng, and S. T. Wu, J. Mater. Chem. 21, 7870 (2011).

${ }^{5}$ Z. Ge, L. Rao, S. Gauza, and S. T. Wu, J. Disp. Technol. 5, 250 (2009).

${ }^{6}$ K. M. Chen, S. Gauza, H. Xianyu, and S. T. Wu, J. Disp. Technol. 6, 49 (2010).

${ }^{7}$ Y. Chen, J. Yan, J. Sun, S. T. Wu, X. Liang, S. H. Liu, P. J. Hsieh, K. L. Cheng, and J. W. Shiu, Appl. Phys. Lett. 99, 201105 (2011).

${ }^{8}$ P. Bos, T. Buzak, and R. Vatne, Proc. SID 26, 157 (1985).

${ }^{9}$ S. Gauza, X. Zhu, W. Piecek, R. Dabrowski, and S. T. Wu, J. Disp. Technol. 3, 250 (2007).

${ }^{10}$ M. Kobayashi, A. Yoshida, and Y. Yoshida, SID Int. Symp. Digest Tech. Papers 41, 1434 (2010).

${ }^{11}$ J. Yan, Y. Li, and S. T. Wu, Opt. Lett. 36, 1404 (2011).

${ }^{12}$ Y. H. Lin, H. S. Chen, H. C. Lin, Y. S. Tsou, H. K. Hsu, and W. Y. Li, Appl. Phys. Lett. 96, 113505 (2010).

${ }^{13}$ Y. Li and S. T. Wu, Opt. Express 19, 8045 (2011).

${ }^{14}$ Z. Ge, S. Gauza, M. Jiao, H. Xianyu, and S. T. Wu, Appl. Phys. Lett. 94, 101104 (2009).

${ }^{15}$ H. J. Coles and S. Morris, Nat. Photonics 4, 676 (2010).

${ }^{16}$ C. D. Tu, C.-L. Lin, J. Yan, Y. Chen, P.-C. Lai, and S.-T. Wu, J. Disp. Technol. 9, 3 (2013).

${ }^{17}$ L. Rao, Z. Ge, S. T. Wu, and S. H. Lee, Appl. Phys. Lett. 95, 231101 (2009).

${ }^{18}$ M. Kim, M. S. Kim, B. G. Kang, M. K. Kim, S. Yoon, S. H. Lee, Z. Ge, L. Rao, S. Gauza, and S. T. Wu, J. Phys. D: Appl. Phys. 42, 235502 (2009).

${ }^{19}$ M. Jiao, Y. Li, and S. T. Wu, Appl. Phys. Lett. 96, 011102 (2010).

${ }^{20}$ H. C. Cheng, J. Yan, T. Ishinabe, and S. T. Wu, Appl. Phys. Lett. 98, 261102 (2011).

${ }^{21}$ L. Rao, J. Yan, S. T. Wu, S. Yamamoto, and Y. Haseba, Appl. Phys. lett. 98, 081109 (2011).

${ }^{22}$ M. Wittek, N. Tanaka, D. Wilkes, M. Bremer, D. Pauluth, J. Canisius, A. Yeh, R. Yan, K. Skjonnemand, and M. Klasen-Memmer, SID Int. Symp. Digest Tech. Papers 43, 25 (2012).

${ }^{23}$ P. R. Gerber, Mol. Cryst. Liq. Cryst. 116, 197 (1985).

${ }^{24}$ J. Yan, Z. Luo, S. T. Wu, J. W. Shiu, Y. C. Lai, K. L. Cheng, S. H. Liu, P. J. Hsieh, and Y. C. Tsai, Appl. Phys. Lett. 102, 011113 (2013). 
${ }^{25}$ L. Rao, J. Yan, S. T. Wu, Y. C. Lai, Y. H. Chiu, H. Y. Chen, C. C. Liang, C. M. Wu, P. J. Hsieh, S. H. Liu, and K. L. Cheng, J. Disp. Technol. 7, 627 (2011).

${ }^{26}$ S. T. Wu, U. Efron, and L. D. Hess, Appl. Opt. 23, 3911 (1984).

${ }^{27}$ C. H. Wen, S. Gauza, and S. T. Wu, Appl. Phys. Lett. 87, 191909 (2005).

${ }^{28}$ S. T. Wu and C. S. Wu, Phys. Rev. A 42, 2219 (1990).

${ }^{29}$ F. C. Frank, Discuss. Faraday Soc. 25, 19 (1958).

${ }^{30}$ H. Xianyu, S. T. Wu, and C. L. Lin, Liq. Cryst. 36, 717 (2009).

${ }^{31}$ L. M. Blinov, Electro-Optical and Magneto-Optical Properties of Liquid Crystals (Wiley, 1983).

${ }^{32}$ Y. Chen, J. Yan, M. Schadt, S. H. Liu, K. L. Cheng, J. W. Shiu, and S. T. Wu, "Diluter Effects on Polymer-Stabilized Blue Phase Liquid Crystals," J. Disp. Technol. (to be published).

${ }^{33}$ T. W. Dakin, in Engineering Dielectrics, edited by R. Bartnikas, R. M. Eichhorn, and B. Brook (ASTM, 1983), Chap. 8, p. 663.
${ }^{34}$ Y. Li, Y. Chen, J. Sun, S. T. Wu, S. H. Liu, P. J. Hsieh, K. L. Cheng, and J. W. Shiu, Appl. Phys. Lett. 99, 181126 (2011).

${ }^{35}$ Y. Liu, Y. F. Lan, H. Zhang, R. Zhu, D. Xu, C. Y. Tsai, J. K. Lu, N. Sugiura, Y. C. Lin, and S. T. Wu, Appl. Phys. Lett. 102, 131102 (2013).

${ }^{36}$ J. Yan, H. C. Cheng, S. Gauza, Y. Li, M. Jiao, L. Rao, and S. T. Wu, Appl. Phys. Lett. 96, 071105 (2010).

${ }^{37}$ J. Yan and S. T. Wu, J. Disp. Technol. 7, 490 (2011).

${ }^{38}$ K. M. Chen, S. Gauza, H. Xianyu, and S. T. Wu, J. Disp. Technol. 6, 318 (2010).

${ }^{39}$ J. Yan, Y. Chen, S. T. Wu, S. H. Liu, K. L. Cheng, and J. W. Shiu, J. Appl. Phys. 111, 063103 (2012).

${ }^{40}$ X. Zhu, Z. Ge, and S. T. Wu, J. Disp. Technol. 2, 2 (2006).

${ }^{41}$ H. C. Cheng, J. Yan, T. Ishinabe, C. H. Lin, K. H. Liu, and S. T. Wu, J. Disp. Technol. 8, 627 (2012). 